\title{
Transfert des polluants nucléaires et conventionnels en rizière irriguée
}

\author{
C. MYTTENAERE (*), R. BITTEL (**), \\ J.-M. MOUSNY (*) et P. DABIN $(*)$
}

(Manuscrit reçu le 28 novembre 1975)

\begin{abstract}
RÉSUMÉ
Les rizières irriguées par submersion constituent, en raison des quantités énormes d'eau utilisées, des écosystèmes particulièrement importants à envisager dans le cadre des recherches sur les conséquences de rejets d'effluents liquides, industriels ou radioactifs, dans les eaux continentales.

On a recouru à des modèles réduits de rizières artificielles, simulant les conditions naturelles de la riziculture en Italie du Nord. On a suivi le comportement de radionucléides de fission ou d'activation, en particulier celui de ${ }^{137} \mathrm{Cs},{ }^{60} \mathrm{Co}$, ${ }^{65} \mathrm{Zn}$, ${ }^{51} \mathrm{Cr}$, et celui de polluants métalliques particulièrement dangereux (Cd, Cr, Sn).

Les phénomènes de transfert et de concentration dans les sols et les divers organes du riz ont été évalués quantitativement et leurs incidences, notamment en ce qui concerne les apports alimentaires à l'homme, discutées.
\end{abstract}

\begin{abstract}
In a general study on the consequences of radioactive and chemical releases in continental waters, flooded rice fields must be considered as a very important ecosystem due to the very large quantities of water used.

In order to approach as much as possible to the natural conditions (irrigated rice fields of Northern Italy) "mini-rice fields" were built and local practices were respected.

The behaviors of activation and fission products $\left({ }^{137} \mathrm{Cs},{ }^{60} \mathrm{Co},{ }^{65} \mathrm{Zn},{ }^{51} \mathrm{Cr}\right.$...) as well as of important heavy metals pollutants $(\mathrm{Cd}, \mathrm{Cr}, \mathrm{Sn})$ were studied and the transfer from water to soil and plant was followed. Concentration factors were calculated for the different organs of the plant and the impact of rice ingested to the dose delivered to man was evaluated.
\end{abstract}

(*) Groupe de Biologie, C.C. E., D. G. Recherche, Science et Éducation, 21020 Ispra, Varese (Italie).

(**) Association EUR-CEA, (DPr), B. P. n 6, 92260 Fontenay-aux-Roses (France). 


\section{INTRODUCTION}

Les eaux continentales servent actuellement d'exutoire aux effluents radioactifs et le cheminement des radionucléides dans les écosystèmes aquatiques a fait l'objet de nombreuses études $[1,4,7,12,13,16,37,38,43,46]$. La multiplication des centrales nucléaires ne fera que renforcer l'acuité des problèmes posés par le rejet d'effluents des installations nucléaires $[1,7,12$, 21] et, dès à présent, sur la base de résultats acquis précédemment, il s'avère nécessaire de chercher des éléments de prévision [44]. De telles' prévisions doivent reposer en partie sur l'étude des voies de transfert, étude qui, dans le cas particulier qui nous intéresse, déborde du cadre de l'environnement plus ou moins immédiat des centrales considérées [11, 14].

L'étude du transfert des radionucléides en rizière irriguée a souligné l'aspect particulier de ce problème [8, 27-29]; de tels résultats sont justifiés partiellement par la modification des caractéristiques édaphiques du sol submergé $[17,35,40,41]$ et par les propriétés physiologiques particulières de la plante de riz $[22,30,31]$. Dans un tel écosystème, l'eau s'est révélée être un facteur très important de transfert de la radiocontamination. D'autre part, les propriétés des eaux d'irrigation peuvent être fortement modifiées par le rejet non contrôlé d'effluents industriels, l'emploi de divers " biocides " et la lixiviation de composés chimiques utilisés en agriculture [43, 46].

La qualité de l'eau d'irrigation a non seulement une grande influence sur le niveau de radiocontamination des plantes [33], mais aussi sur leur teneur en éléments stables.

L'influence des conditions écologiques sur la forme chimique de divers métaux lourds et leur toxicité envers certains organismes aquatiques ont été étudiées par BAUDOIN et ScOPPA [2]. Dans les conditions actuelles, le grand nombre d'interactions entre polluants nucléaires et chimiques présents dans le milieu exige que l'on considère ces derniers comme des polluants associés [3]. Cependant, le transfert des polluants chimiques acheminés par l'eau d'irrigation a été peu étudié et le problème des interactions entre polluants non nucléaires et polluants radioactifs n'a, sans doute, pas encore fait l'objet de toute l'attention désirable [32]. Certaines études montrent que la rizière peut être considérée comme un bassin de décantation et d'épuration des eaux $[10,47,48]$; ce phénomène, favorable en soi en ce qui concerne l'eau, pose le problème de l'accumulation des éléments dans le sol et de leur transfert vers le produit comestible. Les critères d'utilisation des eaux d'irrigation [23] ne tiennent pas compte de ces problèmes et sont, en général, fixés uniquement en fonction des niveaux de toxicité déterminés en conditions contrôlées [20, 23-26, 39].

La présente étude aborde le problème du transfert en rizière irriguée des éléments suivants : césium, cobalt, chrome, zinc, cadmium et étain. Certains isotopes du césium, dont ${ }^{137} \mathrm{Cs}$, sont des produits de fission dont l'importance est bien reconnue; ${ }^{60} \mathrm{Co},{ }^{51} \mathrm{Cr},{ }^{65} \mathrm{Zn}$ sont des produits d'activation figurant dans divers effluents liquides. Parmi les radio-isotopes du cadmium, les plus lourds $(\mathrm{A}=115$ à 119$)$ sont des produits de fission [18]. L'isotope ${ }^{117 \mathrm{~m}} \mathrm{Sn}$ 


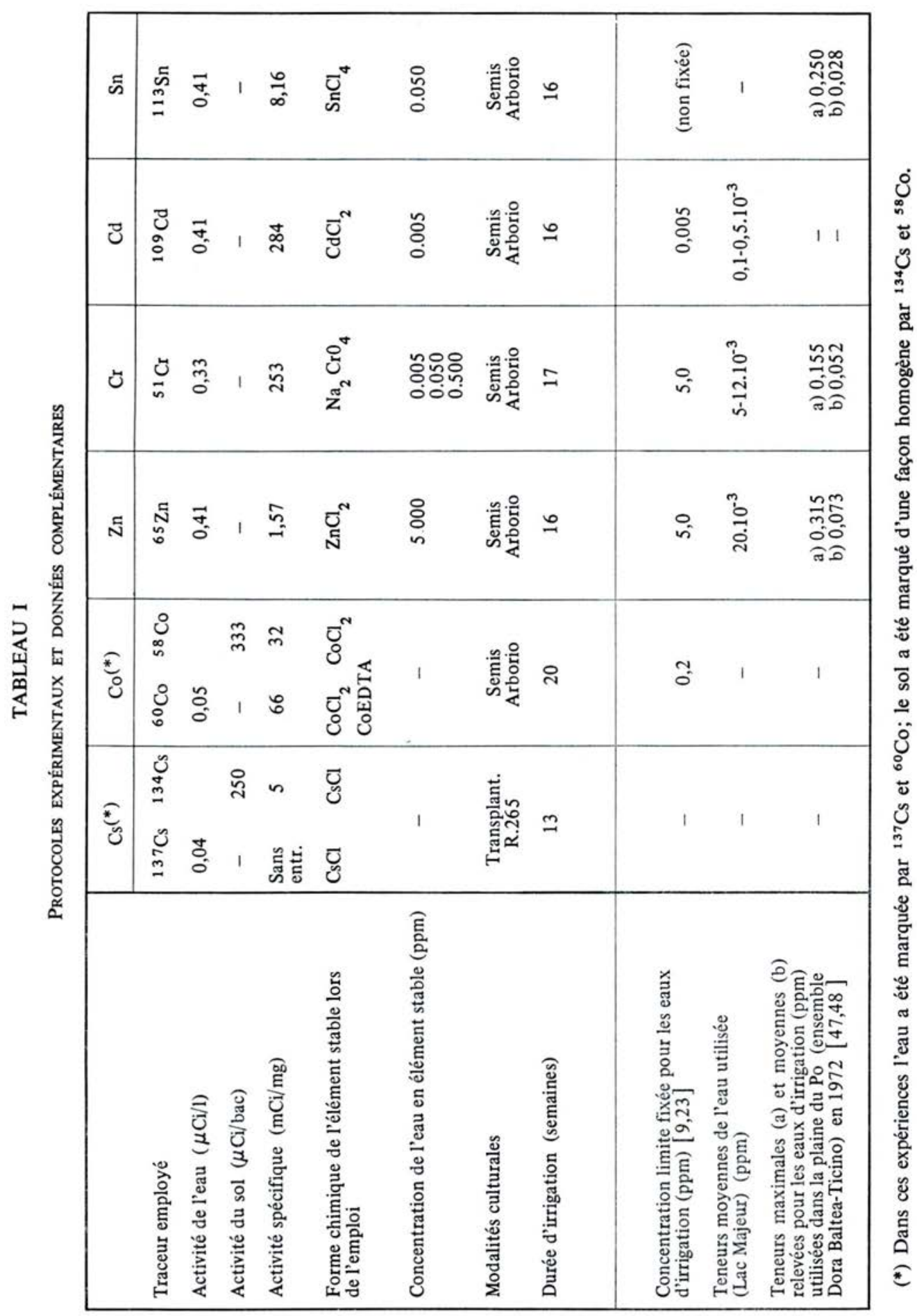


et les isotopes plus lourds de l'étain figurent parmi les produits de fission de l'uranium [18]. Le cadmium doit, en outre, être considéré comme un polluant non nucléaire important $[15,49]$; l'étain est utilisé sous forme de combinaisons complexes comme algocide en début de culture des rizières.

\section{CONDITIONS EXPÉRIMENTALES ET MÉTHODES D'ANALYSE}

Les divers essais ont été réalisés en " modèles de rizière " construits sous serre [28]. Chacun des modèles comprenait sept bacs (0-A-B-C-D-E-F) présentant les dimensions suivantes $0,70 \times 1,00 \times 0,25 \mathrm{~m}$ et placés côte à côte. de façon que l'eau du bac le plus élevé (Bac 0-1501) puisse s'écouler successivement vers les bacs de culture A-B-C-D-E et être recueillie dans le bac F. La vitesse d'écoulement de l'eau a été réglée de telle façon qu'elle s'approche des valeurs observées au champ (1-5 l/ha/s).

Chaque bac de culture a été rempli à l'aide de $125 \mathrm{~kg}$ de sol de rizière prélevé dans la zone rizicole italienne (Vercelli) et dont les caractéristiques sont les suivantes : sable $57 \mathrm{p}$. cent, limon $25 \mathrm{p}$. cent, argile $15 \mathrm{p}$. cent; $\mathrm{N}$ total $1,7 \mathrm{p}$. cent, matière organique $2 \mathrm{p}$. cent; $\mathrm{pH}=5,7$; capacité d'échange : $12,7 \mathrm{meq} / 100 \mathrm{~g}$ sol. Le sol a été fertilisé à l'aide d'un engrais composé à raison de $250 \mathrm{~kg} / \mathrm{ha}$ et le semis (variété Arborio) effectué en poquets (huit poquets par vase). Dès germination, le sol a été recouvert d'une nappe d'eau de $10 \mathrm{~cm}$ et la mise en circulation de l'eau enrichie à l'aide des divers éléments étudiés a été assurée dès le $30^{\circ}$ jour (1501 d'eau par jour pour l'ensemble du modèle). Les éléments étudiés ont été marqués à l'aide des traceurs suivants : ${ }^{134} \mathrm{Cs}$ et ${ }^{137} \mathrm{Cs},{ }^{58} \mathrm{Co}$ et ${ }^{60} \mathrm{Co},{ }^{65} \mathrm{Zn},{ }^{51} \mathrm{Cr},{ }^{109} \mathrm{Cd},{ }^{113} \mathrm{Sn}$. On n'a modifié le mode opératoire général que pour le césium et le cobalt, pour lesquels on a réalisé un double traçage, par ${ }^{137} \mathrm{Cs}$ et ${ }^{60} \mathrm{Co}$ pour l'eau, par ${ }^{134} \mathrm{Cs}$ et ${ }^{58} \mathrm{Co}$ pour le sol.

Le tableau I donne les conditions expérimentales propres aux divers essais. Des échantillons d'eau recouvrant les parcelles expérimentales ont été prélevés journellement; le sol a été échantillonné en début et en fin d'expérience. En fin de culture, les plantes ont été récoltées et séparées en racines, bases des chaumes, chaumes feuillés, panicules vides, balles et caryopses.

La détermination du niveau de radioactivité a été faite par scintillation solide-spectrométrie $\gamma$ et les éléments stables ont été dosés par photométrie en absorption atomique.

\section{RÉSULTATS ET DISCUSSIONS}

\section{TRAnSfert DU CÉsium ET DU COBALT}

On observe (tableau II) un gradient de concentration des racines aux organes les plus éloignés de celles-ci, le caryopse montrant l'activité la plus faible [29]. La méthode du double traçage, utilisée lors de ces essais, montre que la distribution des deux isotopes n'est pas identique. Dans le cas du césium, le rapport des activités ${ }^{137} \mathrm{Cs} /{ }^{134} \mathrm{Cs}$ croît des racines aux graines, 
ce qui indiquerait que le césium 137 apporté par l'eau est plus mobile dans la plante que le césium 134 du sol. C'est le phénomène contraire qui est observé pour le cobalt [6].

TABLEAU II

ACTIVITÉS MOYENNES EN CÉSIUM ET EN COBALT RADIOACTIFS POUR LES DIFFÉRENTS ORGANES DU RIZ IRRIGUÉ (coups/g matière sèche/30 $\mathrm{min}$ )

\begin{tabular}{|l|rrr|rrr|}
\hline \multirow{2}{*}{ Organe } & \multicolumn{3}{|c|}{ Césium } & \multicolumn{3}{c|}{ Cobalt } \\
\cline { 2 - 7 } & $137 \mathrm{Cs}$ & $134 \mathrm{Cs}$ & $137 \mathrm{Cs} / 134 \mathrm{Cs}$ & $60 \mathrm{Co}$ & $58 \mathrm{Co}$ & $60 \mathrm{Co} / 58 \mathrm{Co}$ \\
\hline Racines & 12656 & 4010 & 3,2 & 384089 & 19958 & 19,2 \\
$\begin{array}{l}\text { Parties aériennes } \\
\text { Base des chaumes }\end{array}$ & 20326 & 386 & 52,7 & 183605 & 3950 & 46,5 \\
$\begin{array}{l}\text { Chaumes feuillés } \\
\text { Panicules vides }\end{array}$ & & & & 6085 & 1368 & 4,4 \\
Graines & 13651 & 142 & 96,1 & 371 & 207 & 1,8 \\
Balles & 8772 & 77 & 113,9 & 228 & 149 & 1,5 \\
Caryopses & 4058 & 36 & 112,7 & 89 & 52 & 1,7 \\
\hline
\end{tabular}

$\left({ }^{137} \mathrm{Cs}\right.$ et ${ }^{60} \mathrm{Co}$ proviennent de l'eau, ${ }^{134} \mathrm{Cs}$ et ${ }^{58} \mathrm{Co} \mathrm{du}$ sol).

Des mesures sur le dépôt du cobalt 60 apporté par l'eau d'irrigation sur les sols des rizières montrent que ce radionucléide se fixe sur les quelques premiers centimètres, même s'il est complexé par l'E.D.T.A. (tableau III).

\section{TABLEAU III}

RÉPARTITION DU COBALT 60 APPORTÉ PAR L'EAU D'IRRIGATION DANS LE PROFIL DU SOL EN PLACE, EN POUR CENT DU COBALT TOTAL RETENU PAR LE SOL

\begin{tabular}{|c|c|c|}
\hline Profondeur $(\mathrm{cm})$ & Co non complexé & Co complexé \\
\hline $0-2$ & 92,1 & 61,2 \\
$2-4$ & 3,1 & 26,2 \\
$4-6$ & 1,0 & 11,2 \\
$6-8$ & 1,6 & 1,1 \\
$8-10$ & 2,2 & 0,5 \\
\hline
\end{tabular}

\section{TRANSFERT DU ZINC}

La quantité de radiozinc absorbée par les végétaux représente, dans nos conditions expérimentales, environ 4 p. cent seulement de l'activité apportée par l'eau d'irrigation et retenue dans l'ensemble du "modèle " au cours du cycle cultural.

vol. $10-\mathrm{N}^{\circ} 4$ 


\begin{tabular}{|c|c|c|c|c|c|c|}
\hline & 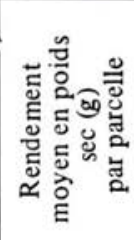 & 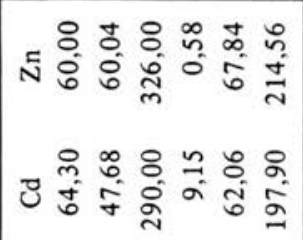 & & & & \\
\hline 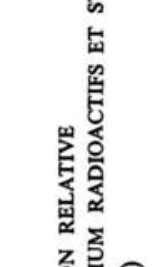 & (ب) & 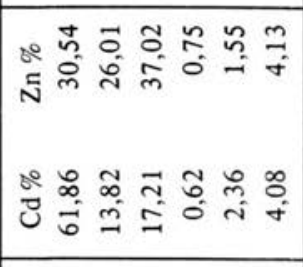 & 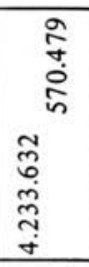 & \begin{tabular}{l}
$\stackrel{\infty}{\sim}$ \\
\multirow{2}{*}{} \\
\multirow{2}{n}{} \\
\end{tabular} & 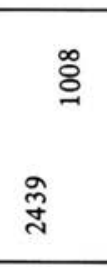 & 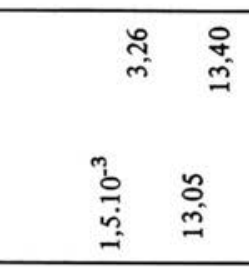 \\
\hline 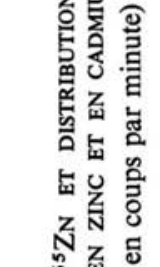 & D & 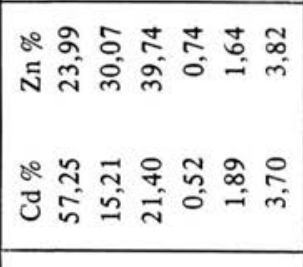 & 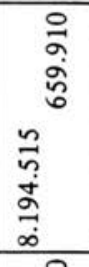 & 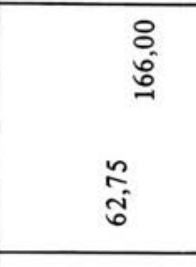 & 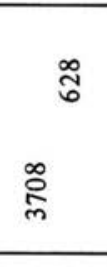 & 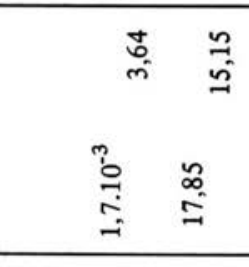 \\
\hline 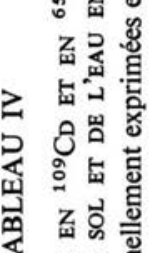 & u & 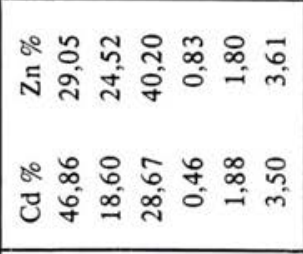 & 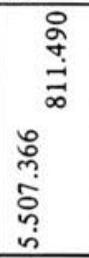 & 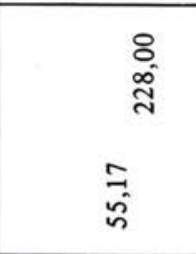 & 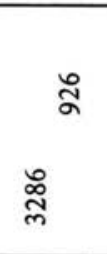 & 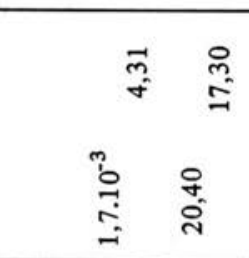 \\
\hline 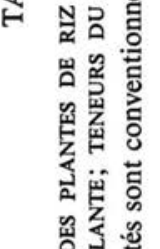 & $\infty$ & 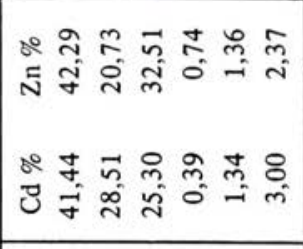 & 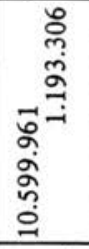 & 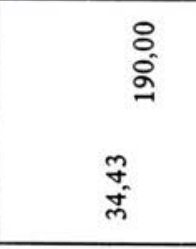 & ळ & 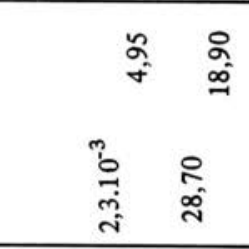 \\
\hline 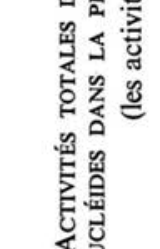 & $\ll$ & 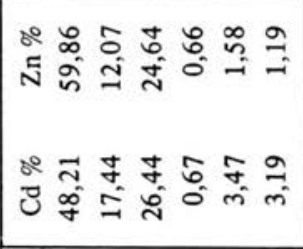 & 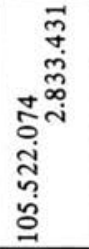 & 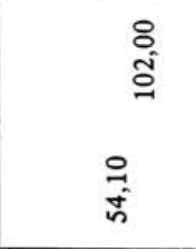 & 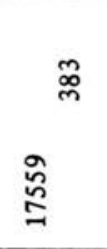 & 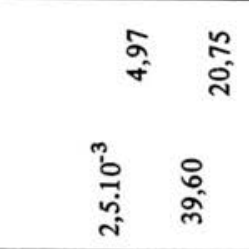 \\
\hline 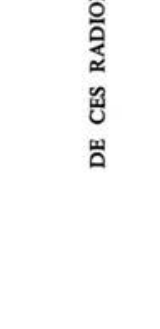 & & 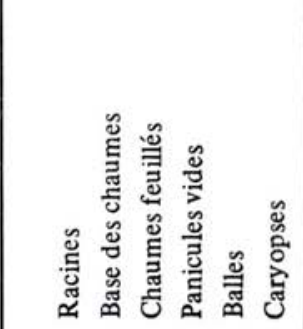 & 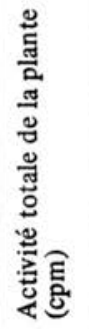 & \multicolumn{3}{|c|}{ 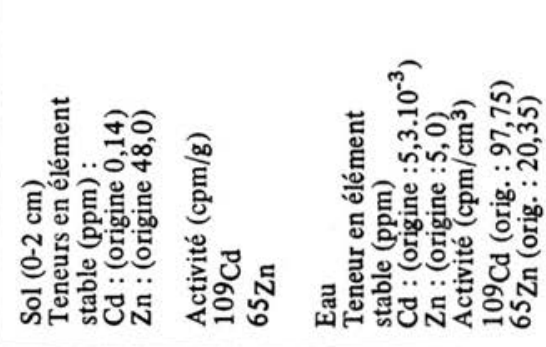 } \\
\hline
\end{tabular}


La couche supérieure du sol (quatre premiers centimètres) a fixé, à elle seule, environ $70 \mathrm{p}$. cent de cette fraction. Ce résultat souligne le pouvoir d'accumulation du sol et les problèmes qu'un tel phénomène pourra poser dans l'avenir.

Le tableau IV donne les teneurs en radiozinc des plantes de riz récoltées à maturité. Le zinc 65 apporté par l'eau d'irrigation, absorbé indirectement (racines profondes) ou directement par la base des chaumes et les racines de surface, se distribue dans toute la plante et l'activité moyenne de la plante ainsi que la distribution du zinc varient d'une parcelle à l'autre de l'écosystème.

En moyenne, les racines contiennent $37 \mathrm{p}$. cent de l'activité totale de la plante alors que le pourcentage attribué au grain de riz blanchi ne s'élève qu'à 3 p. cent. Ces pourcentages varient selon la proximité de la zone des rejets : plus la surface expérimentale est proche du point de rejet (bac A), plus les racines accumulent ${ }^{65} \mathrm{Zn}$ et relativement moins le sol fixe ce radionucléide.

Par conséquent, la compétition entre plante et sol est fonction de la distance qui sépare la source des rejets de la zone expérimentale. En accord avec nos résultats, une forte rétention du zinc 65 par le substrat a été soulignée par des études réalisées aux U.S.A. : rétention de ${ }^{65} \mathrm{Zn}$ par les sédiments de la rivière Columbia [19] et par les boues d'un canal de décharge d'une centrale nucléaire [42].

\section{TABLEAU $\mathrm{V}$}

FACTEURS DE TRANSFERT MOYENS PLANTE/EAU DE LA RIZIÈRe OBTENUS POUR ${ }^{65} \mathrm{ZN}$ ET ${ }^{109} \mathrm{CD}$ Activité plante : $\mathrm{cpm} / \mathrm{g}$ mat. sèche; Activité eau : $\mathrm{cpm} / \mathrm{cm}^{3}$

\begin{tabular}{|l|r|r|}
\hline \multirow{2}{*}{ Organes } & \multicolumn{2}{|c|}{ Facteurs de transfert } \\
\cline { 2 - 3 } & $65_{\mathrm{Zn}}$ & $1^{209} \mathrm{Cd}$ \\
\hline Racines & 421 & 6495 \\
Base des chaumes & 220 & 3064 \\
Chaumes feuillés & 64 & 679 \\
Panicules vides & 48 & 541 \\
Balles & 15 & 340 \\
Caryopses & 8 & 151 \\
\hline
\end{tabular}

Les facteurs de concentration plante-eau d'irrigation sont donnés dans le tableau V. Ces facteurs, établis sur la base des poids secs, varient en moyenne de 421 pour les racines à 8 pour les graines; ces derniers résultats ne fluctuent pas selon la situation de la parcelle expérimentale. De tels chiffres s'accordent avec les résultats publiés par certains chercheurs et repris par l'un de nous pour calculer les doses délivrées à l'homme [5]. L'étude comparative de l'activité spécifique du zinc 65 des organes végétaux et de l'eau d'irrigation montre que les valeurs obtenues pour les plantes sont du même ordre de grandeur que celles calculées pour l'eau (tableau VI). 
Ces observations montrent, par conséquent, que dans nos conditions expérimentales, le zinc absorbé provient surtout de l'eau d'irrigation et très peu du sol. On peut supposer a priori que la quantité totale de zinc stable apportée par l'eau durant toute la culture est grande par rapport au contenu initial du sol. En effet, après 112 jours d'irrigation, l'apport de zinc stable

\section{TABLEAU VI}

ACTIVITÉS SPÉCIFIQUES MOYENNES DE ${ }^{65} \mathrm{ZN}$ ET DE ${ }^{109} \mathrm{CD}$ APPORTÉS PAR L'EAU D'IRRIGATION ET PRÉSENTS DANS LES DIVERS ORGANES VÉGÉTAUX (cpm/ $/ \mathrm{g}$ élément stable)

\begin{tabular}{|c|c|c|c|c|}
\hline Organes & & & & \\
\hline \multirow{2}{*}{ Racines } & \multirow{2}{*}{2,16} & $3,50^{*}$ & \multirow{2}{*}{2125} & 5435 \\
\hline & & 0,94 & & 721 \\
\hline \multirow{2}{*}{ Bases des chaumes } & \multirow{2}{*}{3,31} & 4,16 & \multirow{2}{*}{2206} & 6875 \\
\hline & & 1,86 & & 354 \\
\hline \multirow{2}{*}{ Chaumes feuillés } & \multirow{2}{*}{2,67} & 2,99 & \multirow{2}{*}{882} & 2028 \\
\hline & & 2,11 & & 285 \\
\hline \multirow{2}{*}{ Panicules vides } & \multirow{2}{*}{3,16} & 4,10 & \multirow{2}{*}{754} & 1570 \\
\hline & & 1,28 & & 444 \\
\hline \multirow{2}{*}{ Balles } & \multirow{2}{*}{2,10} & 4,41 & \multirow{2}{*}{565} & 1945 \\
\hline & & 1,07 & & 124 \\
\hline \multirow{2}{*}{ Caryopses } & \multirow{2}{*}{2,73} & 3,59 & \multirow{2}{*}{4777} & 7500 \\
\hline & & 1,51 & & 2826 \\
\hline $\begin{array}{l}\text { Eau d'irrigation } \\
65 \mathrm{Zn}\end{array}$ & \multirow[t]{2}{*}{4,06} & 4,18 & \multirow{2}{*}{11904} & 15840 \\
\hline $\operatorname{Bac} 0=4,12, \operatorname{Bac} 0=18443$ & & 3,82 & & 8700 \\
\hline
\end{tabular}

(*) Valeurs extrêmes obtenues pour les cinq parcelles de l'écosystème.

s'est élevé à $84 \mathrm{~g}$ pour l'ensemble du « modèle " comprenant cinq parcelles de $0,70 \mathrm{~m}^{2}$. Si l'on se base sur la distribution du zinc 65 dans le modèle, $26 \mathrm{~g}$ de zinc stable ont été fixés par $3,50 \mathrm{~m}^{2}$ du sol (épaisseur $4 \mathrm{~cm}$ ) ce qui représente environ $115 \cdot 10^{-6}$ (densité du sol 1,6). Les chiffres donnés dans le tableau VII confirment cet ordre de grandeur. Les résultats qui y sont repris montrent également que le zinc apporté par l'eau d'irrigation augmente fortement le zinc « assimilable » du sol, c'est-à-dire déplaçable par des réactifs chimiques relativement doux. 
TABLEAU VII

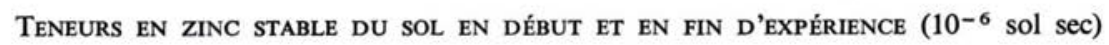

\begin{tabular}{|l|c|c|c|c|c|c|c|}
\hline \multirow{2}{*}{ Réactif d'extraction } & \multirow{2}{*}{ Début d'expérience } & \multicolumn{6}{|c|}{ Fin d'expérience } \\
\cline { 4 - 8 } & & A & B & C & D & E & Moy. \\
\hline Acétate $\mathrm{NH}_{4}(1)$ & 2 & 71 & 84 & 82 & 83 & 132 & 76 \\
\hline $\begin{array}{l}\mathrm{HCl} \mathrm{2N} \mathrm{(2)} \\
\text { (après 1er extract. } \\
\left.\text { Acétate } \mathrm{NH}_{4}\right)\end{array}$ & 53 & 64 & 126 & 150 & 129 & 185 & 131 \\
\hline Total (1)+(2) & 55 & 135 & 210 & 232 & 212 & 317 & 221 \\
\hline $\mathrm{HCl} 2 \mathrm{~N}$ & 48 & 102 & 190 & 228 & 166 & 248 & 187 \\
\hline
\end{tabular}

\section{TRANSFERT DU CADMIUM}

Bien que présent dans l'eau en quantité nettement inférieure au zinc, un faible pourcentage (10 p. cent) du cadmium fixé dans l'écosystème est absorbé par les plantes de riz, le sol jouant surtout le rôle d'accumulateur intermédiaire. La distribution moyenne du cadmium 109 dans la plante diffère très peu de celle du zinc 65 , les racines étant relativement plus riches en ce premier radionucléide (tableau IV). Contrairement au zinc 65, le cadmium 109 en présence d'une très faible teneur en cadmium stable $\left(5.10^{-9}\right)$ se fixe en grande proportion dans le sol du premier bac. Un tel phénomène se traduit par une chute marquée de l'activité des organes végétaux selon la distance parcourue par l'eau. Les facteurs de transfert (tableau V) entre plante (poids sec) et eau sont, par conséquent, plus variables et atteignent, pour les graines, une valeur moyenne plus élevée que pour le zinc (moyenne de 150). Une expérience actuellement en cours a pour but de déterminer, dans les conditions de la rizière irriguée, les facteurs de transfert du cadmium en fonction de sa concentration dans l'eau $\left(0,05 \cdot 10^{-6} ; 0,005 \cdot 10^{-6} ; 0,0025 \cdot 10^{-6}\right)$.

Il semble important de souligner que l'apport de cadmium augmente sensiblement la teneur en zinc des grains de riz (tableau VIII). En effet, la rizière irriguée à l'aide d'eau enrichie en étain peut être considérée comme rizière témoin dans ce cas. Les résultats obtenus soulignent que ce phénomène est dû à une redistribution du zinc absorbé. Un tel phénomène, observé également pour d'autres végétaux, ne trouvera une solution satisfaisante que dans l'étude des formes chimiques du transport du zinc chez les plantes croissant en milieu contenant du cadmium stable [45]. L'activité spécifique du cadmium 109 varie sensiblement d'organe à organe, selon le bac et, en moyenne, est nettement inférieure pour la plante que pour l'eau (tableau VI).

Les valeurs élevées obtenues pour les caryopses (tableau VI) demandent également une étude du mécanisme physiologique responsable du transport et l'identification de la source du cadmium présent dans les caryopses (le cadmium provenant, en grande partie, du pool " eau d'irrigation »).

vol. $10-\mathrm{N}^{\circ} 4$ 
L'activité spécifique plus faible du cadmium présent dans les organes végétaux indiquerait une absorption importante du cadmium stable à partir du sol. Toutefois, l'analyse du sol (Vercelli) avant l'expérience et en fin d'essai a montré que la teneur en cadmium stable (extrait à l'acide chlorhydrique $2 \mathrm{~N}$ ) est passée de 0,14 à $52 \cdot 10^{-6}$. Il est, dès lors, difficile d'invoquer un prélèvement important du cadmium stable présent dans le sol d'origine.

\section{TABLEAU VIII}

TENEURS EN ZINC $\left(10^{-6}\right)$ DES PLANTES RÉCOLTÉES EN MINIRIZIÈRES MODÈLES ENRICHIES EN ZINC ( $5 \mathrm{mg} / 1$ eau d'irrigation), EN CADMiUM ( $5 \mu \mathrm{g} / \mathrm{l}$ eau d'irrigation), ET EN ÉTAIN $(50 \mu \mathrm{g} / 1$ eau d'irrigation)

\begin{tabular}{|l|r|c|c|}
\hline \multirow{2}{*}{} & \multicolumn{3}{|c|}{ Eau enrichie en } \\
\cline { 2 - 4 } & Zn (5 ppm) & Cd $\left(5.10^{-3} \mathrm{ppm}\right)$ & Sn $(0,05 \mathrm{ppm})$ \\
\hline Racines & 5790,40 & 199,84 & 333,92 \\
Bases des chaumes & 1315,80 & 123,14 & 122,18 \\
Chaumes feuillés & 422,08 & 55,18 & 207,64 \\
Panicules vides & 284,20 & 39,38 & 225,88 \\
Balles & 139,04 & 23,66 & 371,42 \\
Caryopses & 52,50 & 90,60 & 25,40 \\
\hline
\end{tabular}

Les données du tableau I montrent, par contre, que la teneur en cadmium stable de l'eau du Lac Majeur peut atteindre $10 \mathrm{p}$. cent de la teneur en cadmium stable apporté $\left(0,5 \cdot 10^{-9}-5 \cdot 10^{-9}\right)$. Les résultats obtenus pourraient donc être attribués à un marquage défectueux du cadmium de l'eau par les ${ }^{109} \mathrm{Cd}+\mathrm{Cd}$ stable introduits; un tel résultat pourrait, par conséquent, être imputé à un manque d'identité entre les formes chimiques introduite et naturelle de l'eau. Si cette interprétation peut justifier la différence observée entre eau et plante, elle ne peut être invoquée pour expliquer les différences observées entre les organes de la partie aérienne ne baignant pas dans l'eau (chaumes feuillés et grains), à moins que le cadmium des graines ne provienne préférentiellement d'un compartiment déterminé de l'écosystème. Un manque d'équilibre isotopique au sein de la plante a déjà été observé, dans les mêmes conditions expérimentales, pour le césium [31].

\section{TRANSFERT DU CHROME}

Le radiochrome apporté par l'eau d'irrigation sous forme de chromate se concentre surtout au niveau radiculaire et dans la base des chaumes. Aucune activité n'a été décelée dans les graines. En rizière irriguée, et sous cette forme chimique, le radiochrome ne présente donc pas de problème du point de vue de la radioprotection (tableau IX). Ces résultats confirment ceux obtenus en conditions contrôlées [34]; le chrome apporté sous forme d'ions $\mathrm{Cr}^{3+}$ ou $\mathrm{CrO}_{4}^{2-}$ est fixé surtout par les constituants des parois cellulaires, et très peu de chrome est transporté des racines vers la partie aérienne. Un tel transport 


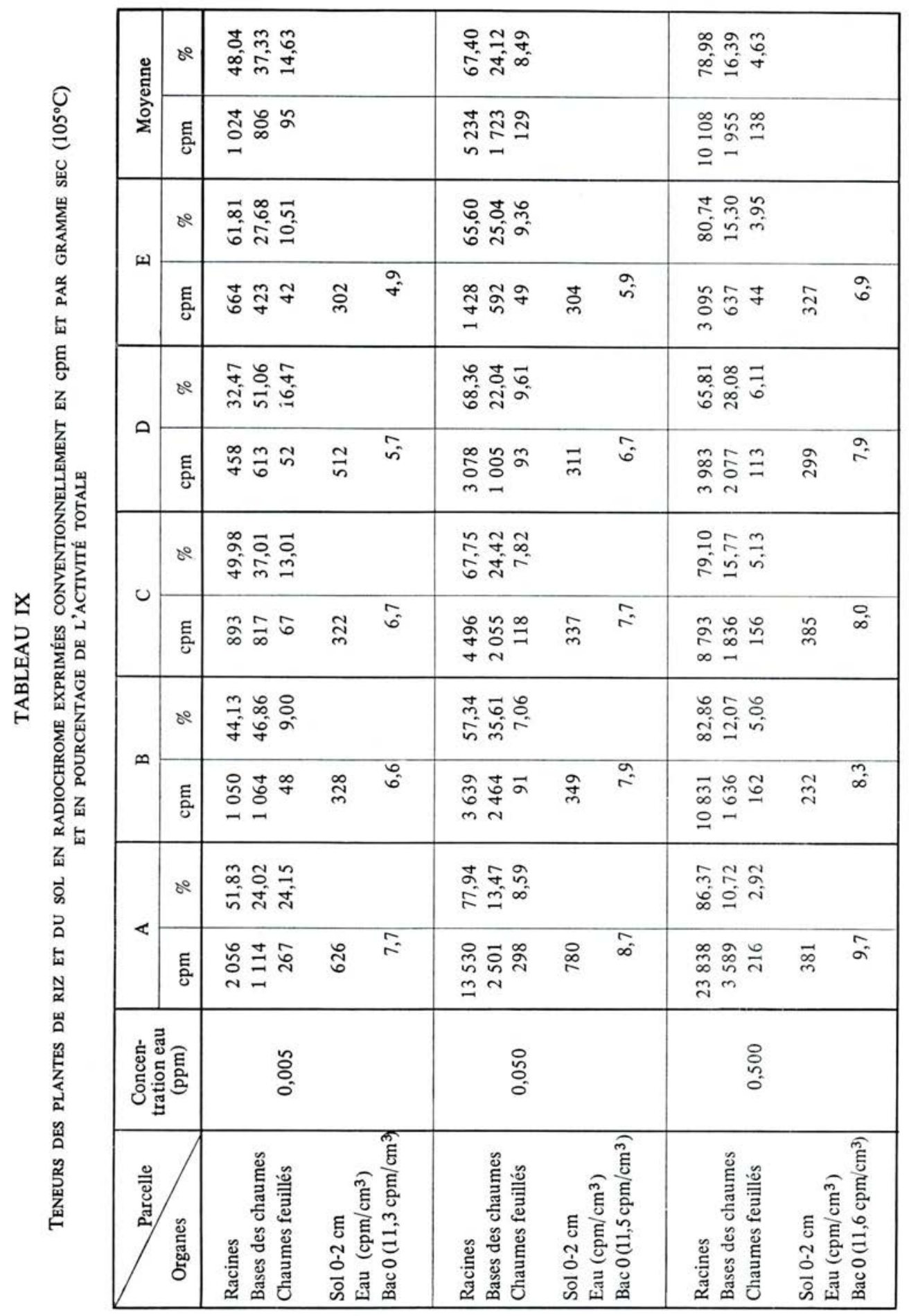


ne pourrait être accru que par suite d'une chélation du chrome présent dans le milieu; toutefois, dans ce cas, la quantité absorbée par les plantes est fortement réduite. Il est intéressant de souligner l'influence de la concentration du chrome stable sur la radiocontamination en ${ }^{51} \mathrm{Cr}$ des racines et des bases des chaumes. Bien que l'activité en ${ }^{51} \mathrm{Cr}$ introduite soit la même pour les trois concentrations, la teneur en ${ }^{51} \mathrm{Cr}$ des plantes irriguées à l'aide d'une eau contenant la plus faible concentration en chrome stable $\left(5.10^{-9}\right)$ est la plus faible. Dans ce cas, il est possible de tenir le même raisonnement que

\section{TABLEAU $\mathrm{X}$}

Teneurs des PLANTES ET DU SOL EN RADioÉtain

(exprimées conventionnellement en cpm et par gramme $\sec \left(105^{\circ} \mathrm{C}\right)$ et en pourcentage de l'activité totale)

\begin{tabular}{|c|c|c|c|c|c|c|c|c|c|c|c|}
\hline \multirow{2}{*}{ Organes } & \multicolumn{2}{|c|}{ A } & \multicolumn{2}{|c|}{ B } & \multicolumn{2}{|c|}{ C } & \multicolumn{2}{|c|}{ D } & \multicolumn{2}{|c|}{ E } & \multirow{2}{*}{$\underset{\%}{\text { Moyenne }}$} \\
\hline & opm & $\%$ & cpm & $\%$ & cpm & $\%$ & cpm & $\%$ & $\mathrm{cpm}$ & $\%$ & \\
\hline Racines & 875 & 44,22 & 276 & 33,38 & 209 & 37,16 & 95 & 40,35 & 69 & 42,82 & 39,59 \\
\hline Bases chaumes & 960 & 30,28 & 694 & 52,83 & 578 & 55,08 & 264 & 55,42 & 131 & 42,60 & 47,24 \\
\hline Chaumes feuillés & 154 & 25,51 & 34 & 13,78 & 14 & 7,75 & 4 & 4,22 & 9 & 14,58 & 13,17 \\
\hline Panicules vides & - & & & & & & & & & & \\
\hline Balles & - & & & & & & & & & & \\
\hline Caryopses & - & & & & & & & & & & \\
\hline Sol $(0-2 \mathrm{~cm})$ & 3088 & & 1537 & & 906 & & 421 & & 211 & & \\
\hline $\begin{array}{l}\text { Eau Bac } 0 \\
\left(\mathrm{cpm} / \mathrm{cm}^{3}: 7,4\right.\end{array}$ & 3,30 & & 3,20 & & 3,05 & & 2,80 & & 2,70 & & \\
\hline
\end{tabular}

pour le cadmium stable. En effet, la teneur en chrome stable des eaux du Lac est de 5 à $12 \cdot 10^{-9}$ et les quantités apportées artificiellement de 5,50 et $500 \cdot 10^{-9}$. Si l'on suppose que les formes chimiques des chromes naturel et introduit sont différentes (absence d'équilibre isotopique), les plantes du premier traitement vivent dans un milieu contenant la moitié de chrome non marqué, présent sans doute sous forme de complexe. La situation inverse est vraie pour les plantes recevant $500 \cdot 10^{-9}$ de chrome. Si ce raisonnement s'avère valable, et sur la base des résultats acquis en conditions contrôlées, la translocation relative devrait être supérieure dans le premier cas. Le tableau IX nous montre, en effet, que plus de $14 \mathrm{p}$. cent du chrome se trouvent dans les feuilles pour un apport de $5 \cdot 10^{-9}$ de chrome alors que ce pourcentage n'est que de $5 \mathrm{p}$. cent environ dans le cas du dernier traitement. L'intensité de la translocation vers la partie aérienne pourrait, dans le cas du chrome, être utilisée comme indicateur de la forme chimique du chrome présent dans le milieu. 


\section{Transfert de l'Étain}

Avant toute interprétation des résultats, il est nécessaire de faire remarquer que l'expérimentation a porté sur l'étain déversé dans le milieu à l'état salin, alors que les algocides utilisés en riziculture sont à base d'étain organométallique.

Dans nos conditions expérimentales, les résultats obtenus montrent que l'étain ne doit pas retenir l'attention du nutritioniste : en effet, aucune trace d'activité en ${ }^{113} \mathrm{Sn}$ n'a été décelée dans les caryopses (tableau X), racines et bases des chaumes étant les deux organes les plus riches en étain. Ces résultats montrent, de plus, que si à brève échéance, l'irrigation de la rizière ne présente pas. de problème du point de vue de la pollution en étain, il n'en est pas de même à long terme. En effet, la culture n'exporte qu'une faible quantité de l'élément apporté $(0,43 \mathrm{p}$. cent de la quantité fixée dans le modèle, cette dernière représentant $68 \mathrm{p}$. cent du total apporté en cours de culture). La presque totalité de cet élément est donc fixée par le sol et des travaux ultérieurs devraient être consacrés à l'étude des taux de fixation, d'assimilabilité et de lixiviation en fonction du temps.

\section{CONCLUSIONS}

Les résultats obtenus montrent que certains éléments sont intensément retenus par les végétaux, mais que les transferts vers les organes consommés par l'homme ne sont quantitativement importants, parmi les éléments étudiés, que pour les isotopes du cadmium, du césium et du zinc. Pour ces trois éléments, il peut donc a priori se poser des problèmes de protection dus à l'ingestion de riz par l'homme :

- dans le cas éventuel de pollutions radioactives;

- dans le cas éventuel de pollutions chimiques.

On peut, par un calcul classique [50], évaluer le débit de dose délivrée à l'organe critique de l'homme du fait de l'ingestion de $1 \mathrm{~kg}$ de riz, quand l'activité spécifique de l'eau d'irrigation est prise égale à $1 \mathrm{pCi} / \mathrm{kg}$ ou 1 (tableau XI).

En admettant qu'un radionucléide $j$ parvienne à l'organe de référence de l'homme à raison de $A$ unités d'activité par unité de temps et en soit éliminé selon une loi exponentielle simple, si $q$ est l'activité du radionucléide présent à l'instant $t$ dans l'organe, si $\lambda$ est la constante de décroissance effective dans l'organe :

$$
\frac{d q}{d t}+\lambda q=A,
$$

d'où, si $q=0$ pour $t=0$,

$$
q=\frac{A}{\lambda}\left(1-e^{-\lambda t}\right) .
$$

vol. $10-\mathrm{N}^{\circ} 4$ 


\section{TABLEAU XI}

DÉBIT DE DOSE DÉLIVRÉE A L'ORGANE CRITIQUE DE L'HOMME POUR UNE INGESTION ANNUELLE DE $1 \mathrm{~kg}$ DE RIZ, L'EAU D'IRRIGATION CONTENANT 1 pCi/l DE RADIONUCLÉIDE

\begin{tabular}{|c|c|c|c|c|c|c|c|}
\hline Radionucléide & Organe critique & $\mathrm{m}(\mathrm{kg})$ & $F$ & fe & $\epsilon(\mathrm{MeV})$ & $\lambda\left(a^{-1}\right)$ & $D(\mu \mathrm{rem} / \mathrm{an})$ \\
\hline${ }^{137} \mathrm{Cs}$ & $\begin{array}{l}\text { organisme } \\
\text { entier }\end{array}$ & 70 & 20 & 1 & 0,6 & 3,6 & 1 \\
\hline${ }^{60} \mathrm{Co}$ & $»$ & 70 & 0,6 & 0,3 & 1,5 & 27 & 0,003 \\
\hline $65 \mathrm{Zn}$ & $»$ & 70 & 8 & 0,1 & 0,3 & 1,3 & 0,05 \\
\hline $115 \mathrm{~m}_{\mathrm{Cd}}$ & & & & & & & \\
\hline (déduit de & 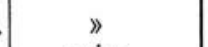 & 70 & 150 & 0,1 & 0,6 & & 0,01 \\
\hline $\begin{array}{l}\text { résultats } \\
\left.\text { sur }{ }^{109} \mathrm{Cd}\right)\end{array}$ & reins & 0,3 & 150 & $2,5.10^{-4}$ & 0,6 & 6 & 0,2 \\
\hline $\begin{array}{l}125_{\mathrm{Sn}} \text { et } \\
51_{\mathrm{Cr}}\end{array}$ & - & - & - & - & - & - & $\begin{array}{l}\text { négligeable } \\
\left(\leqslant 10^{-10}\right)\end{array}$ \\
\hline
\end{tabular}

Pour un radionucléide $j$ et un vecteur alimentaire $i, A$ s'exprime par le produit de la quantité $Q$ du vecteur alimentaire ingéré par l'homme en l'unité de temps, de l'activité $y$ par unité de poids de l'aliment et de la fraction $f_{e}$ du radionucléide ingéré qui parvient à l'organe critique de l'homme

$$
A=Q y f_{e},
$$

d'où, en portant dans (1) :

$$
q=\frac{Q y f_{e}}{\lambda}\left(1-e^{-\lambda t}\right) .
$$

Si $m$ est le poids de l'organe critique, son activité spécifique sera $: q / m$. La dose délivrée $D$, entre les instants $\mathrm{O}$ et $t$, à l'organe critique peut être mesurée par le produit, à un facteur $k$ près dépendant des unités,

- de l'activité spécifique de l'organe $q / m$;

- de l'énergie effective par désintégration $\varepsilon$;

- du nombre de désintégrations par unité d'activité :

$$
D=k \frac{q}{m} \cdot \varepsilon
$$

En portant la valeur de $q$ dans (4), on obtient :

$$
D=k \frac{Q y f_{e} \varepsilon}{m} \frac{1-e^{-\lambda t}}{\lambda} .
$$


Si $t$ est grand, $1-e^{-\lambda t} \approx 1$ et $D$ s'écrit

$$
D=k \frac{Q y f_{e} \varepsilon}{m \lambda} \text {. }
$$

D'après la définition du facteur de transfert $F$ du radionucléide $j$ de l'eau au vecteur alimentaire $i$ :

$$
\frac{y}{x}=F ; \quad y=F x
$$

$x$ étant l'activité spécifique de l'eau. En portant (7) dans (6) :

$$
D=k \cdot \frac{F Q \varepsilon f_{e}}{m \lambda} \cdot x
$$

Si on exprime $D$ en rem.an ${ }^{-1}, Q$ en $\mathrm{g} . \mathrm{an}^{-1}, m$ en $\mathrm{g}, \varepsilon$ en $\mathrm{MeV}, \lambda$ en an $^{-1}$, $x$ en pCi.g $\mathrm{g}^{-1}$ :

$$
k=18,7 \cdot 10^{-3} \text {. }
$$

Le tableau XI donne les valeurs de $D$ en $\mu$ rem/an pour $x=10^{-3} \mathrm{pCi} \cdot \mathrm{g}^{-1}$ (1 pCi. $\left.1^{-1}\right)$ et $Q=1000 \mathrm{~g} . \mathrm{an}^{-1}\left(1 \mathrm{~kg} \cdot \mathrm{an}^{-1}\right)$.

Pour des activités spécifiques de l'ordre de 10 à $100 \mathrm{pCi}$ par litre d'eau d'irrigation et des quantités consommées de riz de l'ordre de $10 \mathrm{~kg}$ par an, les débits de dose seraient de l'ordre de 100 à $1000 \mu \mathrm{rem} / \mathrm{an}$ pour ${ }^{137} \mathrm{Cs}$, 0,3 à $3 \mu \mathrm{rem} / \mathrm{an}$ pour ${ }^{60} \mathrm{Co}$, de 5 à $50 \mu \mathrm{rem} / \mathrm{an}$ pour ${ }^{65} \mathrm{Zn}$, de 20 à $200 \mu \mathrm{rem} / \mathrm{an}$ pour ${ }^{115 \mathrm{~m}} \mathrm{Cd}$ (si on transpose les données du ${ }^{109 \mathrm{~m}} \mathrm{Cd} \mathrm{au}{ }^{115} \mathrm{Cd}$ qui est un produit de fission), c'est-à-dire qu'ils seraient, au plus, égaux au millirem par an. Pour les radio-isotopes du chrome et de l'étain, on est très au-dessous de ces valeurs. Cependant, il reste un problème non résolu pour l'étain, celui résultant de l'utilisation de complexes organométalliques qui sont les formes physicochimiques utilisées dans les pesticides. Il n'est, par ailleurs, pas exclu que, pour certains de ces éléments, en particulier pour ${ }^{137} \mathrm{Cs},{ }^{65} \mathrm{Zn}$ et peut-être pour des radio-isotopes du cadmium. des problèmes de radioprotection résultant de la consommation du riz se posent, du fait de l'additivité des effets de rejets provenant de sites nucléaires voisins polluant indirectement un même réseau d'irrigation. Enfin, en raison du nombre d'individus qui peuvent être exposés du fait d'une contamination du riz, il est a priori impossible de négliger les doses résultant de l'ingestion du riz, même si celles-ci sont relativement faibles.

En outre, la rétention des radionucléides par l'horizon superficiel des sols pose, dès maintenant, le problème de la radioprotection des travailleurs opérant sur des rizières en dehors des périodes de submersion (inhalation de poussières).

L'aspect le plus directement critique est assurément celui qui résulte de la pollution chimique [51]. Il faut, en particulier, souligner la valeur très élevée du facteur $F$ de transfert eau-grain pour le cadmium. Si on admet la valeur

VOL. $10-\mathrm{N}^{\circ} 4$ 
de 150 pour $F$, une consommation de $10 \mathrm{~kg}$ de riz par an et par individu entraîne l'ingestion de $5.10^{-3} \times 150 \times 10$ soit $7,5 \mathrm{mg}$ de cadmium par an si, dans l'eau d'irrigation, il y a $5 \cdot 10^{-3} \mathrm{mg}$ de cadmium par litre, concentration de tolérance admise généralement pour l'eau d'irrigation en irrigation intense. Ceci représente environ $20 \mu \mathrm{g}$ par jour, ce qui est de l'ordre de la quantité moyenne ingérée par l'homme.

Il est clair que la pratique de l'irrigation par submersion tend à enrichir en cadmium les horizons superficiels du sol, si l'eau d'irrigation est polluée. Ceci crée un problème potentiel pour les cultures qui pourraient, après plusieurs années, succéder au riz sur les mêmes sols.

\section{BIBLIOGRAPHIE}

[1] Antonucci L., Bramati L., Fiorentino F., Malossi S. Management experience with radioactive wastes from ENEL nuclear power stations. In Management of low- and intermediate-level radioactive wastes, Aix-en-Provence, 7-11 septembre 1970, Vienne, AIEA, 1970, 261-274.

[2] Baudouin M. F., Scoppa P. The influence of environmental factors on the toxicity of heavy metals to aquatic organisms, CEC Joint research Centre, Ispra, Italy, Annual Report 1973.

[3] BItTel R. Les pollutions associées aux pollutions radioactives. Radioprotection, 1974, 9, 111-130.

[4] Bittel R. Transferts de la pollution radioactive des sources de contamination jusqu'à l'homme. Agriculture, 1973, 366, 167-169.

[5] Bittel R., Merlini M., Berg A., Myttenaere C., Ravera O., Verot J. L., VAN DER BORGHT O. Étude du transfert à l'homme par ses aliments des radio-isotopes du ruthénium, du cobalt et du zinc rejetés dans les eaux continentales. La radioécologie appliquée à la protection de l'homme et de son environnement. Rome, 7-10 septembre 1971. Commission des Communautés européennes. Luxembourg, EUR-4800, 2, 869.

[6] Bittel R., Merlini M., Myttenaere C., Ravera O. Études des paramètres de transfert du radiocobalt dans les écosystèmes continentaux aquatiques et irrigués. Rapports CEA-R 4157, EUR 4628 f, 1971.

[7] Blomeke J. O., Harrington F. E., Waste management of nuclear power stations. Nuclear Safety, 1968, 9, 239-248.

[8] Bourdeau P., Cavalloro R., Myttenaere C., Verfaillie G. Movement of fallout radionuclides in irrigated ecosystems of the Po Valley, Italy. Health Physics, 1965, 11, 1429-1444.

[9] Cesaretti C. M. Inquinamento ed agricoltura. ISVET, 1970, 30, 1-136.

[10] Corbetta G. Consequenze dell' impiego di acque inquinate in risaia. Tribuna Agricola, 1972, 12, 4.

[11] Coulon R. Études effectuées lors de l'implantation d'installations nucléaires. Agriculture, 1973, 366, 184-186.

[12] De Bortoli M., GAGlione P. Sorveglianza del sito all' interno del CCR EURATOM, ISPRA. Rapport EUR-4051 i, 1968.

[13] de Bortoli M., Gaglione P., Myttenaere C. Radioiodine transfer in an irrigated grassland ecosystem (Marcita). Giorn. Fis. Sanit. Prot. Rad., 1972, 16, 184-190.

[14] Delmas J., Grauby A. L'irrigation, voie de transfert de la radiopollution, une étude radioécologique continentale. Agriculture, 1973, 366, 178-180. 
[15] Friberg L. M. D., Piscator M. D., Nordberg G. M. B. Cadmium in the environment, Cleveland, C.R.C. Press, 1971.

[16] Gloyna E. F. Transport of radionuclides in a model aquatic system. In Symp. Intern. Radioécologie. Cadarache, 8-12 septembre 1969, 3-32.

[17] Giordano P. M., Mortvedt J. J. Rice response to zinc in flooded and non flooded soil. Agron. J., 1972, 64, 521-524.

[18] Haissinsky M., Adloff J. P. Dictionnaire radiochimique des éléments, Genève, Sadési, 1968.

[19] Jennings C. D., Osterberg C. H. Sediment radioactivity in the Columbia river estuary. In Proceedings of the second national symposium on radioecology. Ann. Arbor, Michigan, May 15-17, 1967, 300-306.

[20] Juste C. Actions toxiques des oligo-éléments. Ann. Agron., 1970, 2, 549-571.

[21] KAN B. Radionuclides in the environment at nuclear power stations. In Proc. 3 national Symposium on radioecology, Oak Ridge, Tennessee, May 10-12, 1971, 30-36.

[22] IshizuKa Y. Physiology of the rice plant. Adv. Agron., 1971, 22, 241-315.

[23] LA Noce T. Criteri di qualità delle acque usate in agricoltura per irrigazione. ISSET, 1970, 30, 137-175.

[24] Lindsay W. L. Zinc in soils and plant nutrition. Adv. Agron., 1972, 24, 147-186.

[25] Lisk D. J. Trace metals in soils, plants and animals. Adv. Agron., 1972, 24, 267-325.

[26] Lotri G. Lo zinco nel metabolismo vegetale. In Simposio Internazionale di agrochimica sulla fitonutrizione oligominerale, Punta Ala, 2-6 Ottobre 1972. Pisa, Istituto di industrie agrarie dell' Università degli Studi.

[27] Myttenaere C., Bourdeau P. Transfert des radionucléides en région irriguée. Giorn. Fis. Sanit. Prot. Rad., 1968, 12, 179-181.

[28] Myttenaere C., Bourdeau P. Quelques aspects des problèmes de la radiocontamination des cultures irriguées. In Actes du Congrès Int. sur la Radioprotection du milieu, Toulouse, 15-16 mars 1967. Société française de Radioprotection, 289-305.

[29] Myttenaere C., Bourdeau P., Bittel R. Importance relative de l'eau et du sol dans la contamination indirecte en radiocésium et radiocobalt des rizières irriguées. In Environmental contamination by radioactive materials, Vienne, 24-28 mars 1969. AIEA, Vienne, 1969, 175-182.

[30] Myttenaere C., Bourdeau P., Masset M. Relative importance of soil and water in the indirect contamination of flooded rice with radiocesium. Health Physics, 1969, 16, 701-707.

[31] Myttenaere C. Absorption of radiocesium by flooded rice : relative importance of roots and shoot base in the transfer of radioactivity. Plant Soil, 1972, 36, 215-218.

[32] Myttenaere C. Radioécologie et pollution conventionnelle. Bull. Ass. Ing. Fac. Sc. Agron., Louvain, 1973, 11-13.

[33] Myttenaere C., Mousny J. M. Influence de la qualité de l'eau d'irrigation sur le transfert du radiocésium et du radiostrontium en rizière irriguée. In La Radioécologie appliquée à la protection de l'homme et de son environnement, Rome, 7-10 septembre 1971, Luxembourg, Commission des Communautés européennes, EUR-4800, 1972, 2, 1255-1275.

[34] Mytrenaere C., Mousny J. M. The distribution of chromium-51 in lowland rice in relation to the chemical form and to the amount of stable chromium in the nutrient solution. Plant Soil, 1974, 41, 65-72.

[35] Myttenaere C., Markwordt U. Influence of water regime on the indirect absorption of radiocesium, radiostrontium and radiocobalt by lowland rice. In Proc. 3rd National Symposium on radioecology, Oak Ridge, May 10-12, 1971, 212-217.

vol. $10-\mathrm{N}^{\circ} 4$ 
[36] Myttenaere C., Masset M. Availability of stable manganese and ${ }^{54} \mathrm{Mn}$ to the rice plant in flooded soil. Soil Sci., 1969, 107, 296-301.

[37] Picat P., Grauby A. Évolution de la radiocontamination des canaux d'irrigation alimentés par le Rhône. In Symposium International de Radioécologie, C.E.A., Cadarache, 8-12 septembre 1969, 33-81.

[38] Picat P., Bovard P., Grauby A., Ijuin M., Reveillault J., Faure J., Andres J. Bilan de la dispersion des radionucléides dans un bassin hydrobiologique. Perspectives en fonction des activités nucléaires : usines de traitement et réacteur de puissance. In La radioécologie appliquée à la protection de l'homme et de son environnement, Rome, 7-10 septembre 1971. Luxembourg, Commission des Communautés européennes, EUR-4800, 1972, 1, 139-174.

[39] Perigaud S. Liaisons carentielles entre sols, végétaux et animaux. Ann. Nutr. Alim., 1971, 25, 327-378.

[40] Ponnamperuma F. N. The chemistry of submerged soils. Adv. Agron., 1972, 24, 29-88.

[41] Ponnamperuma F. N. Dynamic aspects of flooded soils and the nutrition of the rice plant. Proc. Symposium International Rice Research Institute, 1965, 295-328.

[42] Salo E. O., Leet L. W. The concentration of ${ }^{65} \mathrm{Zn}$ by oysters maintained in the discharge canal of a nuclear power plant. In Proceedings of the 2 nd national symposium on radioecology. Ann. Arbor, Michigan, May 15-17, 1967, 363-371.

[43] Stemple R. Chromate pollution of water - detection effects and prevention, a bibliography. Report ORNL-TM-3450, 1971.

[44] Strauch S. The year 2000 - nuclear power and man. In Proc. 3rd National Symposium on radioecology, Oak Ridge, Tennessee, May 10-12, 1971, 53-62.

[45] TURNER M. A. Effect of cadmium treatment on cadmium and zinc uptake by selected vegetable species. J. exp. Qual., 1973, 2, 118-119.

[46] Watson D. G., Cushing C. E., Coutant C. C., Templeton W. L. Effect of Hanford reactor shutdown on Columbia river biota. Biol. Dept. Battelle Memorial Inst., Pacif. Northwest Lab., Richland, Washington, 1966, 8.

[47] EnTE NAZIonALE Risi. Inquinamento dei canali d'irrigazione e di scolo delle risaie del compensario Dora Baltea-Ticino. I. Caratteristiche fisicochimiche delle acque, 1973, 39.

[48] ENTE Nazionale Risi. Inquinamento dei canali d'irrigazione e di scolo delle risaie del Vercellese, 1971, 36.

[49] Identification and control of pollutants of broad international significance, United Nations Conference on the human environment, Stockholm, 5-16 June 1972.

[50] Commission Internationale de Protection Radiologique (CIPR). Rapport du comité II sur la dose admissible en cas d'irradiation. Publication CIPR 2, Paris, Gauthier-Villars, 1963.

[51] US Department of Interior. Water quality criteria, 1968. 